\title{
Characteristics and clinical significance of lymph node metastases near the recurrent laryngeal nerve from thoracic esophageal carcinoma
}

\author{
K. Ye, J.H. Xu, Y.F. Sun, J.A. Lin and Z.G. Zheng \\ Department of Oncology, \\ The Second Affiliated Hospital of Fujian Medical University, \\ Quanzhou, Fujian Province, China \\ Corresponding authors: K. Ye / J.H. Xu \\ E-mail: yekai72@126.com / jianhua78123abc@163.com
}

Genet. Mol. Res. 13 (3): 6411-6419 (2014)

Received January 9, 2014

Accepted May 5, 2014

Published August 25, 2014

DOI http://dx.doi.org/10.4238/2014.August.25.4

\begin{abstract}
This study aimed to evaluate the characteristics of lymph node (LN) metastases from thoracic esophageal carcinoma near the recurrent laryngeal nerve and the influence of these metastases on patient prognosis and to determine the reasonable regional $\mathrm{LN}$ dissection range. The clinical data from 120 patients who underwent resection for thoracic esophageal carcinoma were analyzed retrospectively. LN metastases near the recurrent laryngeal nerve were detected in $34.2 \%$ of the cases, and the metastasis rates in the left and right LNs near the recurrent laryngeal nerve were 20.8 and $15.8 \%$, respectively. The primary tumor site (metastasis rates for esophageal cancer in the upper thoracic segment $v s$ chest or lower thoracic segment: $60.0 v s$ 40.3 or $15.8 \%$, respectively; $\mathrm{P}<0.01$ ), tumor differentiation (poorly differentiated $v s$ well differentiated or differentiated: $56.0 v s 22.0$ or $35.6 \%$, respectively; $\mathrm{P}<0.05$ ), and tumor invasion depth (T3 and $\mathrm{T} 4$ vs $\mathrm{T} 1$ and $\mathrm{T} 2$ : 42.9 and $50.0 \%$ vs 8.33 and $14.3 \%$, respectively; $\mathrm{P}<$ 0.01 ) were factors that significantly influenced LN metastasis near the
\end{abstract}


recurrent laryngeal nerve $\mathrm{LN}$ metastases near the recurrent laryngeal nerve were associated with cervical LN metastasis. The 3-year survival rate of patients with LN metastasis near the recurrent laryngeal nerve was much lower than that of patients with other LN metastases (29.3 vs $58.2 \%$; $\mathrm{P}<0.05)$. In thoracic esophageal carcinoma cases, LNs near the recurrent laryngeal nerve should be resected. This could improve the patient prognosis and reduce the incidence of postoperative local recurrence.

Key words: Esophageal neoplasm; Recurrent laryngeal nerve; Lymphadenectomy; Lymph node; Metastasis

\section{INTRODUCTION}

Esophageal cancers are strongly invasive and malignant tumors, and lymph node (LN) metastasis is a crucial factor for staging these tumors and the prognosis associated with surgical treatment. LN metastasis of esophageal cancer exhibits the downward, upward and skip spread characteristics. Esophageal cancer has a predilection for metastasis to the recurrent laryngeal nerve LNs on both sides, which is a vital yet difficult LN dissection point. Therefore, to evaluate the characteristics of para-recurrent laryngeal nerve LN metastases from thoracic esophageal carcinomas and the influence of these metastases on patient prognosis and to identify a reasonable and effective dissection range, complete data from 120 patients with esophageal cancer who underwent bilateral recurrent laryngeal nerve $L N$ dissection via 3 incisions (cervix-chest-abdomen) in our department between January 2000 and December 2004 were retrospectively analyzed.

\section{MATERIAL AND METHODS}

\section{Clinical data}

A total of 120 patients who underwent radical surgery to treat esophageal cancers of the right chest in our department between January 2000 and December 2004 were studied. Among these patients, 76 were men and 44 were women, resulting in a male:female ratio of 1.73:1. The patients' ages ranged from 35 to 75 years with a mean age of $58 \pm 3.6$ years. According to the Union for International Cancer Control (UICC) 1997 grading standard, the invasion sites included the upper chest in 15 patients, middle chest in 67 patients, and lower chest in 38 patients. According to the UICC 1997 staging standard and on the basis of the depth of tumor invasion, 12 patients had stage T1 tumors, 28 patients had stage T2 tumors, 56 patients had stage T3 tumors, and 24 patients had stage T4 tumors. After removal, the tumor length was measured along the longitudinal axis of the invading esophageal tumor, considering a standard of 2 to $10 \mathrm{~cm}$. The mean tumor length was $4.7 \pm 2.5 \mathrm{~cm}$; there were 67 patients with tumors $\leq 5 \mathrm{~cm}$ and 53 patients with tumors $>5 \mathrm{~cm}$. With regard to the degree of tumor differentiation, the tumors were well differentiated in 50 patients, differentiated in 45 patients, and poorly differentiated in 25 patients. All patients' diagnoses were pathologically confirmed as squamous cell carcinoma. 


\section{Inclusion criteria}

The patients of the Department of Oncology at the Second Affiliated Hospital of Fujian Medical University, Quanzhou, China, were selected to undergo esophagectomy through the right chest for subtotal resection and dissection of LNs in the neck, chest, and abdomen (R0 resection) between January 2000 and December 2004. Pathological examination of the dissected specimens confirmed that the total number of metastatic LNs was $>15 ; 66$ patients with esophageal cancer did not have distant metastasis. Complete clinical data and follow-up results were available for all patients after excluding those with esophageal cancer who received preoperative radiotherapy/chemotherapy. During the evaluation period, a total of 624 patients underwent esophagectomy. As the surgical trauma associated with the Akiyama operation is severe, the patients in this study were younger than patients evaluated in other studies because younger patients exhibited better physical conditions.

\section{Surgical methods}

All the patients underwent subtotal esophageal excision via the right chest and 3-field LN dissection including both sides of the neck, chest, and abdomen (Akiyama et al., 1994). The fifth rib was cutted off, and the chest was opened by making a right posterolateral incision. The esophagus was resected and removed from the thoracic cavity. The mediastinal LNs in the chest were dissected in all patients. The patients were placed in a supine position after closure of the thoracic cavity, and concomitantly subjected to LN dissection including the neck and abdomen. A collar-shaped incision was made in the neck, and a median incision was made in the abdomen, after which the LNs were cleared from both sites. The stomach was subsequently freed and shaped into a tube that was then placed in the neck near the esophageal bed and the portion of the esophagus in the neck, and attached via line end-to-side anastomosis.

The right recurrent laryngeal nerve LN was removed as follows: the airway behind the mediastinal pleura was opened to expose the right subclavian artery, followed by the blunt dissection of the right recurrent laryngeal nerve through the vagus nerve trunk at the level of the right subclavian artery. The blood vessel upstream of the tracheoesophageal groove was subsequently bypassed after exposing an approximately 1-mm thick section of the nerve. The surrounding LNs and fatty tissues were subsequently removed.

The LNs around the left recurrent laryngeal nerve were removed as follows: the left recurrent laryngeal nerve was revealed around the aortic arch and moved upward to the left tracheoesophageal groove. The esophagus was revealed to be at the top of the free pleura and subsequently pulled to the dorsal side, while an assistant pulled the trachea to the ventral side. Careful blunt dissection was performed, slightly behind and to the left of the trachea for easy exposure of approximately $1 \mathrm{~mm}$ of the left recurrent laryngeal nerve, to allow the separation of the nerve root from the neck upward and the removal of the surrounding LNs and fatty tissues.

\section{Clinical follow-up and statistical analysis}

Eight patients (6.8\%) were lost to follow-up. The patients lost to follow-up after 3 visits were considered dead. The time to death for the patients lost to follow-up was calculated on the basis of the last follow-up date; all other patients had a follow-up period of 3 years. Chest 
and neck computed tomography was performed every 6 months to evaluate the status of local recurrence or metastasis. The data were analyzed by the SPSS 11.5 software package (SPSS, Inc., Chicago, IL, USA). Numerical data were analyzed using the chi-squared test. Values are reported as means \pm standard deviations unless otherwise indicated. Survival rates were calculated according to the life-table method; survival curve comparisons were performed using the log-rank test. $\mathrm{P}$ values $<0.05$ were considered to be significant.

\section{RESULTS}

\section{LN metastasis}

Among the 120 patients with esophageal cancer, 41 experienced recurrent laryngeal nerve LN metastasis (34.2\%). A total of 720 recurrent laryngeal nerve LNs were detected, and a mean of $6.8 \mathrm{LNs}$ were dissected; 168 metastatic LNs were detected, and the rate of LN metastasis was $23.4 \%$. The rate of right recurrent laryngeal nerve LN metastasis was $20.8 \%$ $(25 / 120)$, which was slightly higher than the rate of left recurrent laryngeal nerve LN metastasis $(15.8 \%, 19 / 120)$; the rate of skip metastasis to the recurrent laryngeal nerve LNs was $4.2 \%(5 / 120)$.

\section{Relationships between recurrent laryngeal nerve LN metastasis and clinical patho- logical factors}

The recurrent laryngeal nerve $\mathrm{LN}$ metastasis rate $(66.7 \%)$ was significantly higher $(\mathrm{P}$ $<0.01)$ in patients with upper thoracic segment esophageal cancer than in patients with chest $(40.3 \%)$ or lower thoracic segment esophageal cancer $(15.8 \%)$, suggesting that the region affected by esophageal cancer correlated significantly with recurrent laryngeal nerve LN metastasis. Furthermore, the rates of recurrent laryngeal nerve LN metastasis were significantly higher in patients with T3 and T4 esophageal cancer (42.9 and 50.0\%, respectively) than in patients with $\mathrm{T} 1$ and $\mathrm{T} 2$ esophageal cancer $(8.33$ and $14.3 \%$, respectively; $\mathrm{P}<0.01)$, suggesting deeper tumor invasion correlated with an increased risk of recurrent laryngeal nerve LN metastasis. The rate of recurrent laryngeal nerve LN metastasis was also significantly higher $(\mathrm{P}<0.05)$ in patients with poorly differentiated esophageal cancer $(56.0 \%)$ than in patients with well-differentiated (22.0\%) and differentiated esophageal cancers (35.6\%), suggesting that esophageal cancer differentiation correlated closely with the rate of recurrent laryngeal nerve LN metastasis. In this study, the patients were divided into 2 groups according to the length of the esophageal cancer lesions $(\leq 5 \mathrm{or}>5 \mathrm{~cm})$. The results revealed a lack of significant correlation between the rate of recurrent laryngeal nerve LN metastasis and the length of the esophageal lesion $(\mathrm{P}>0.05$; Table 1$)$.

\section{Relationship between recurrent laryngeal nerve LN and cervical LN metastasis}

Among the patients with esophageal cancer, the rate of LN metastasis near the recurrent laryngeal nerve in patients with cervical LN metastasis was $51.2 \%(21 / 41)$, which was significantly higher than the rate in patients without cervical LN metastasis $(13.9 \%, 11 / 79$; $\mathrm{P}<0.01)$. 


\begin{tabular}{|c|c|c|c|c|c|}
\hline Groups & Case & Positive case & Negative case & $\chi^{2}$ value & $P$ value \\
\hline Age (years) & 120 & 41 & 79 & 0.010 & $>0.05$ \\
\hline$<55$ & 49 & 17 & 32 & & \\
\hline$\geq 55$ & 71 & 24 & 47 & & \\
\hline Gender & & & & 0.660 & $>0.05$ \\
\hline Male & 76 & 28 & 48 & & \\
\hline Female & 44 & 13 & 31 & & \\
\hline Tumor site & & & & 14.312 & $<0.01$ \\
\hline Upper chest & 15 & 9 & 6 & & \\
\hline Chest section & 67 & 27 & 40 & & \\
\hline Lower thoracic & 38 & 6 & 32 & & \\
\hline Tumor length (cm) & & & & 2.275 & $>0.05$ \\
\hline$\leq 5 \mathrm{~cm}$ & 67 & 19 & 48 & & \\
\hline$>5 \mathrm{~cm}$ & 53 & 22 & 31 & & \\
\hline Invasion depth & & & & 12.943 & $<0.01$ \\
\hline $\mathrm{T}_{1}$ & 12 & 1 & 11 & & \\
\hline $\mathrm{T}_{2}$ & 28 & 4 & 24 & & \\
\hline $\mathrm{T}_{3}^{2}$ & 56 & 24 & 32 & & \\
\hline $\mathrm{T}_{4}^{3}$ & 24 & 12 & 12 & & \\
\hline Differentiate agree & & & & 8.632 & $<0.05$ \\
\hline Well-differentiation & 50 & 11 & 39 & & \\
\hline Differentiation & 45 & 16 & 29 & & \\
\hline Poor-differentiation & 25 & 14 & 11 & & \\
\hline Cervical lymph node metastasis & & & & 19.595 & $<0.01$ \\
\hline+ & 32 & 21 & 11 & & \\
\hline - & 88 & 20 & 68 & & \\
\hline
\end{tabular}

\section{Postoperative complications}

The incidence of recurrent laryngeal nerve injury was $12.5 \%(15 / 120)$, and the incidence of cervical anastomotic fistula was $8.33 \%(10 / 120)$. The incidence of pulmonary infection was $20.8 \%(25 / 120)$; among patients with pulmonary infection, the incidence was higher in the recurrent laryngeal nerve injury group $(40 \%, 6 / 15)$ than in the non-recurrent laryngeal nerve injury group $(18.1 \%, 19 / 105)$. Two patients $(2.5 \%)$ died of respiratory failure perioperatively.

\section{Recurrence rate after surgery and survival}

The local recurrence rate of all the patients at 3 years after surgery was $8.3 \%$. This rate for patients who did not undergo 3-field LN dissection in cases of esophageal cancer was $18 \%$ during the same period. Of all the patients, the survival rate 3 years after surgery was $48.3 \%(58 / 120)$. The 3-year survival rates of patients with and without recurrent laryngeal LN metastasis of esophageal cancer were $29.3 \%(12 / 41)$ and $58.2 \%(46 / 79)$, respectively. This difference was significant $(\mathrm{P}<0.05)$.

\section{DISCUSSION}

LN metastasis is the most common manifestation of esophageal cancer metastasis. The esophageal submuscosa is rich in dense lymphatic vessels, vertical and horizontal vessels and the mediastinal lymph network communicate with each other. In addition to continuous diffusion along the vessel walls, the cancer cells can also metastasize through the mucosal 
collateral lymphatic vessels to pass through the muscle layer and the theca externa, and finally result in skip distant metastasis away from the primary tumor. Accordingly, LN metastasis exhibits both specificity and complexity. The patient prognosis was generally poor after esophageal cancer resection. LN metastasis in the upper mediastinum and cervical region is the main cause of local recurrences of esophageal cancer (Altorki et al., 2002). LNs near the recurrent laryngeal nerve on both sides are the most common sites for LN metastasis and account for a major proportion of the mediastinal LNs between the mediastinal septum and the cervical region. Clinically, patients with esophageal cancer present with hoarseness primarily because of the compression caused by the metastatic LNs in the tracheal esophageal groove. These findings help in the selection of a surgical approach, dissection of intraoperative LNs, and determination of the postoperative treatment in order to facilitate discussions of the role of LN metastasis near the recurrent laryngeal nerve on both sides in patients with esophageal cancer. Fujita et al. (2002) reported on LN metastasis and recurrence in 70 patients with esophageal cancer who had undergone 3-field LN dissection and found that the rate of right recurrent laryngeal nerve LN metastasis was the highest, although the most common site of LN recurrence was the left recurrent laryngeal nerve LNs. The data presented by Zhilian et al. (1998) indicated that the rate of bilateral recurrent laryngeal nerve LN metastasis was 39.6\%; the upper mediastinal LNs in the neck were affected in $91.8 \%$ of the cases. In this study, the rate of LN metastasis near the bilateral recurrent laryngeal nerve was $34.2 \% ; 15.8 \%$ involving the left LNs and $20.8 \%$ involving the right LNs. The rate of skip metastasis to the LNs near the recurrent laryngeal nerve was $4.2 \%(5 / 120)$. This finding suggests that esophageal cancer has a predilection for metastasis to the LNs near the recurrent laryngeal nerve on both sides; therefore, all patients with esophageal cancer in the thoracic segment should undergo dissection of the LNs near the bilateral recurrent laryngeal nerve. This might reduce errors in clinical disease staging and increase the number of dissected LNs in order to achieve R0 resection.

$\mathrm{LN}$ metastasis is among the important factors affecting the prognosis of patients with esophageal cancer. Tabira et al. (1999) reported that not only the number of LNs but also the LN sites, particularly those near the recurrent laryngeal nerve, were prognostic indicators. Baba et al. (1994) reported that the local recurrence rates increased along with an increase in the number of metastatic LNs, suggesting that LN metastasis near the recurrent laryngeal nerve was the strongest predictor of prognosis. Three-field LN dissection, particularly LN dissection near the recurrent laryngeal nerve, can effectively improve the life span of patients with esophageal cancer. Malassagne et al. (1997) reported that the 5-year survival rate of patients with LN metastasis near the recurrent laryngeal nerve was $21 \%$, a significantly lower rate than that of patients with non-LN metastasis (47\%). Therefore, LN metastasis near the recurrent laryngeal nerve is an important factor that affects the prognosis of patients with esophageal cancer.

The postoperative follow-up data showed that the 3-year survival rate of patients with LN metastasis near the recurrent laryngeal nerve was $29.3 \%$, a significantly lower rate than that observed in patients without recurrent laryngeal nerve LN metastasis (58.2\%). This finding suggests that LN metastasis near the recurrent laryngeal nerve was an important factor affecting the postoperative survival rate of patients with esophageal cancer. Local recurrence and distant metastasis are important modes of postoperative failure. Among these, recurrence in the upper mediastinum and cervical region is the most common pattern of recurrence. Altorki et al. (2002) found that the local recurrence rate following traditional radical resection for esophageal cancer was 20 to $60 \%$; in contrast, the local recurrence rate of the patients 
who underwent 3-field LN dissection was only $8 \%$. In this study, the rate of recurrence in the upper mediastinum and neck was $8.3 \%$, which was significantly lower than the rate reported in patients who underwent conventional cancer resection. These results were similar to those reported in the literature.

During dissection, the LNs near the recurrent laryngeal nerve are localized in the narrow area of the cervicothoracic junction and at the intersection of the mediastinum and cervical LNs. Therefore, we considered the LNs near the recurrent laryngeal nerve to be the vital regional LNs of patients with esophageal cancer. The dissection of LNs near the recurrent laryngeal nerve could reduce the rate of local esophageal cancer recurrence and improve the survival rate, and thus facilitate the formulation of postoperative treatment plans. This procedure could also help to determine whether patients require postoperative radiotherapy and chemotherapy in the cervical region and mediastinum.

In this study, the main factors affecting LN metastasis near the recurrent laryngeal nerve in patients with esophageal cancer included the primary tumor location, tumor invasion depth, and tumor differentiation. The rates of recurrent laryngeal nerve LN metastasis from tumors in the upper, middle, and lower thoracic regions were 66.7, 40.3, and 15.8\%, respectively. These differences were found to be significant $(\mathrm{P}<0.01)$. This finding suggests that closer the lesion to the proximal end of the recurrent laryngeal nerve, higher is the risk of LN metastasis near recurrent laryngeal nerve. Although LNs at the thoracic esophagus and the left and lesser curvatures of the stomach are common metastatic sites, skip metastasis was also observed in the LNs near the recurrent laryngeal nerve. The rates of LN metastasis near the recurrent laryngeal nerve in patients with well, moderately, and poorly differentiated esophageal cancers were 22.0, 35.6, and 56.0\%, respectively. The rates of LN metastasis near the recurrent laryngeal nerve in patients with $\mathrm{T} 1, \mathrm{~T} 2, \mathrm{~T} 3$, and $\mathrm{T} 4$ stage disease were $8.3,14.3$, 42.9 , and $50.0 \%$, respectively, suggesting that esophageal cancer differentiation and the depth of tumor invasion were significantly correlated with recurrent laryngeal nerve LN metastasis $(\mathrm{P}<0.05)$. A lower degree of esophageal cell differentiation, deeper tumor invasion depth, and increased probability of LN metastasis near the recurrent laryngeal nerve increased the difficulty of achieving complete surgical resection.

Compared to the usual treatment of the same type of esophageal cancer via a right anterior lateral or left chest incision, the patients in this study were treated via an incision along the posterolateral right chest through which the bilateral recurrent laryngeal nerve could be dissected under direct vision, and the nearby LNs could be sharply dissected. Common postoperative complications included recurrent laryngeal nerve injury and pulmonary infection. In the literature, $12-20 \%$ patients experienced recurrent laryngeal nerve injuries; these were mostly unilateral. In the present study, the incidence of recurrent laryngeal nerve injury was $7.8 \%$. These injuries generally occurred during the initial stages of the adoption of this surgical method, the time at which injury rates were higher. After the surgeons became skilled and familiar with the anatomical features and techniques, the rates of recurrent laryngeal nerve injury, postoperative complications, and mortality decreased.

Additionally, the incidence of pulmonary infection in the group with recurrent laryngeal nerve injuries was $40 \%(6 / 15)$, a higher rate than that observed in the group without recurrent laryngeal nerve injuries $(18.1 \%, 19 / 105)$. The patients could not close the glottis completely because of the recurrent laryngeal nerve injury, which resulted in pulmonary infection leading to weak expectoration, shortness of breath, and retention of secretion causing 
pulmonary infection as well as respiratory failure leading to digestive juice aspiration and related chemical burns in the respiratory passages. Peng et al. (2006) evaluated the causes of pulmonary infection associated with cervical and upper thoracic esophageal cancers, and found that pulmonary infections in patients with recurrent laryngeal nerve injuries accounted for $68.4 \%$ of all cases of infection; the authors concluded that recurrent laryngeal nerve injury was the main cause of pulmonary infection. Preventing recurrent laryngeal nerve injuries could effectively reduce the incidence of pulmonary infection. In our experience, the patients were treated with timely bedside bronchoscopy suction, and tracheotomy, if necessary.

Both local and international studies have reported (Zhilian et al., 1998; Fujita et al., 2002; Feng et al., 2005) LN metastasis rates of thoracic esophageal cancer ranging from 16 to $43 \%$ and recurrence rates ranging from 6 to $16 \%$; therefore, it is important to predict and detect LN metastases of esophageal cancer in order to improve the efficacy of esophageal cancer treatment. Tabira et al. (2000) found that LN metastasis near the recurrent laryngeal nerve in patients with esophageal cancer correlated significantly with cervical LN metastasis and that LN metastasis near the recurrent laryngeal nerve was predictive of cervical LN metastasis. Yoshioka et al. (2002) reviewed 71 patients with esophageal cancer who underwent regular 3 -field LN dissection and found a rate of cervical LN metastasis of $51.6 \%$ in patients with $\mathrm{LN}$ metastases near the recurrent laryngeal nerve; in contrast, the cervical LN metastasis rate of patients without LN metastases near the recurrent laryngeal nerve was significantly lower at $11.6 \%$. A multivariate analysis revealed that LN metastasis near the recurrent laryngeal nerve was a strong predictor of cervical LN metastasis. The need for cervical LN dissection for thoracic esophageal cancer remains controversial. Shiozaki et al. (2001) reported that, among patients with LN metastases near the recurrent laryngeal nerve, the life span of those patients who had undergone 3-field LN dissection was higher than that of patients who had undergone 2 -field LN dissection. However, in patients with non-LN metastases near the recurrent laryngeal nerve, the life span of patients who underwent 3-field and 2-field lymphadenectomy was similar. Ueda et al. (2006) used a reverse-transcription polymerase chain reaction method to determine which patients with esophageal cancer and LN metastases near the bilateral recurrent laryngeal nerve would require neck dissection. When patients who underwent traditional 3-field LN dissection were compared with patients who were found intraoperatively to have recurrent laryngeal nerve LN metastases but had not undergone cervical LN dissection, the follow-up data revealed similar rates of cervical LN recurrence in both groups (2.6 vs 6.7\%); however, the 3-year survival rate of the former group of patients was $83.3 \%$, which was higher than that of the latter $(57.2 \%)$. This suggested that intraoperative monitoring of the recurrent laryngeal nerve facilitates decisions regarding the necessity of cervical LN dissection. This study found that in patients with thoracic esophageal cancer, the rate of LN metastasis near the recurrent laryngeal nerve was $51.2 \%$, which was significantly higher than the rate of non-LN metastasis near the recurrent laryngeal nerve. We consider that in patients with esophageal cancer, the LNs near the recurrent laryngeal nerve represent a critical route of mediastinal LN metastasis; therefore, recurrent laryngeal nerve LNs could be considered the "sentinel" LNs in patients with cervical esophageal cancer.

In conclusion, we recommend that all patients with thoracic esophageal cancer undergo dissection of the LNs near the recurrent laryngeal nerve on both sides regardless of the tumor location, size, and depth of invasion; we believe that this will improve the radical cure rate, definite staging, and life span while reducing the local recurrence rate. The detection of 
LNs near the recurrent laryngeal nerve will help to identify those patients with esophageal cancer who require cervical LN dissection.

\section{ACKNOWLEDGMENTS}

Research supported by the funding (\#JB12091 and \#JB11051) from the Fujian Education Department.

\section{REFERENCES}

Akiyama H, Tsurumaru M, Udagawa H and Kajiyama Y (1994). Radical lymph node dissection for cancer of the thoracic esophagus. Ann. Surg. 220: 364-372.

Altorki N, Kent M, Ferrara C and Port J (2002). Three-field lymph node dissection for squamous cell and adenocarcinoma of the esophagus. Ann. Surg. 236: 177-183.

Baba M, Aikou T, Yoshinaka H, Natsugoe S, et al. (1994). Long-term results of subtotal esophagectomy with three-field lymphadenectomy for carcinoma of the thoracic esophagus. Ann. Surg. 219: 310-316.

Feng QL, Shang SY and Zhao XJ (2005). The study on metastasis of lymph node for carcinoma of the thoracic esophagus. Chin. J. Clin. Oncol. 32: 706-708.

Fujita H, Sueyoshi S, Tanaka T and Shirouzu K (2002). Three-field dissection for squamous cell carcinoma in the thoracic esophagus. Ann. Thorac. Cardiovasc. Surg. 8: 328-335.

Malassagne B, Tiret E, Duprez D, Coste J, et al. (1997). Prognostic value of thoracic recurrent nerve nodal involvement in esophageal squamous cell carcinoma. J. Am. Coll. Surg. 185: 244-249.

Peng CL, Dong XP and Zhao XG (2006). Postoperative pneumonia due to cervical part and upper thoracic part esophageal carcinoma surgery: retrospective analysis. Chin. J. Nosocomiol. 16: 1248-1249.

Shiozaki H, Yano M, Tsujinaka T, Inoue M, et al. (2001). Lymph node metastasis along the recurrent nerve chain is an indication for cervical lymph node dissection in thoracic esophageal cancer. Dis. Esophagus 14: 191-196.

Tabira Y, Kitamura N, Yoshioka M, Tanaka M, et al. (1999). Significance of three-field lymphadenectomy for carcinoma of the thoracic esophagus based on depth of tumor infiltration, lymph nodal involvement and survival rate. $J$. Cardiovasc. Surg. 40: 737-740.

Tabira Y, Yasunaga M, Tanaka M, Nakano K, et al. (2000). Recurrent nerve nodal involvement is associated with cervical nodal metastasis in thoracic esophageal carcinoma. J. Am. Coll. Surg. 191: 232-237.

Ueda Y, Shiozaki A, Itoi H, Okamoto K, et al. (2006). Intraoperative pathological investigation of recurrent nerve nodal metastasis can guide the decision whether to perform cervical lymph node dissection in thoracic esophageal cancer. Oncol. Rep. 16: 1061-1066.

Yoshioka S, Fujiwara Y, Sugita Y, Okada Y, et al. (2002). Real-time rapid reverse transcriptase-polymerase chain reaction for intraoperative diagnosis of lymph node micrometastasis: clinical application for cervical lymph node dissection in esophageal cancers. Surgery 132: 34-40.

Zhilian S, Kunsou Z and Suoyan L (1998). The investigation of thoracic esophageal cancer neck and upper mediastinal lymph node metastasis. Chin. J. Clin. Oncol. 53: 530-532. 